\title{
Optical measurement techniques for mobile and large-scale dimensional metrology
}

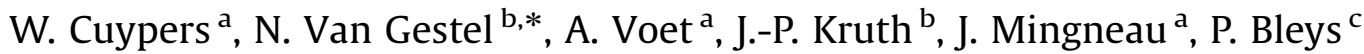 \\ a De Nayer Instituut, J. De Nayerlaan 5, 2860 Sint Katelijne Waver, Belgium \\ ${ }^{\mathrm{b}}$ Department of Mechanical Engineering, K.U. Leuven, Celestijnenlaan 300b, 3001 Leuven, Belgium \\ ${ }^{\mathrm{c}}$ WTCM, Celestijnenlaan 300c, 3001 Leuven, Belgium
}

\section{A R T I C L E I N F O}

Available online 5 May 2008

Keywords:

Mobile CMM

Portable CMM

Large-scale metrology

Photogrammetry

\begin{abstract}
A B S T R A C T
Dimensional inspection tasks are often carried out on conventional coordinate measuring machines (CMMs). These CMMs can differ in layout and size depending on the application area. They can have measurement volumes up to $100 \mathrm{~m}^{3}$. However, when measuring large objects it is not always possible to bring a large object to these conventional CMMs. That is why for these applications mobile measuring systems are an ideal solution. These systems often measure the dimensions through optical techniques, like interferometry and optical triangulation. After a short survey of common optical measurement techniques for mobile and large-scale measurements, this paper focuses on two industrial cases where different techniques were used to solve a measurement problem. The first case covers the measurement of a large iron casting with an optical LED-based triangulation system. It was possible to predict problems that would occur with the machining of the part, like unfinished surfaces after milling due to material shortage, and adapt the alignment of the part to prevent these problems. The second case covers the measurement of a double-decker train by means of photogrammetry, as an alternative for the currently used total station. Despite some specific drawbacks of photogrammetry systems it was possible to obtain the same accuracy and to reduce the overall inspection time significantly in comparison with the current situation.
\end{abstract}

(c) 2008 Elsevier Ltd. All rights reserved.

\section{Introduction}

Accuracy specifications are becoming more and more demanding, also for large objects and product assemblies, like large castings (gear boxes, etc.), large welding constructions (trains, etc.), and large plastic objects (bumpers, etc.). Conventional coordinate measuring machines (CMMs) are often not appropriate in these cases. Measurement volumes of these CMMs can be too small; further, it is often not possible or unwanted to bring the part for inspection to the CMM. Therefore most large-scale measuring equipments are mobile.

Only 3D-coordinate measurement systems are discussed in this paper. Optical measuring systems for specific purposes like level, angle, and straightness measurements are not considered. In fact, the enhanced accuracy of general purpose mobile $3 \mathrm{D}$-coordinate measuring machines makes it possible to replace in some cases specific measurement systems for level, angle, and straightness measurements.

Almost all CMMs for large-scale applications rely on optical position measurement in contrast to conventional CMMs that are

\footnotetext{
* Corresponding author. Tel.: +32 16322537; fax: +3216322987.

E-mail address: nick.vangestel@mech.kuleuven.be (N. Van Gestel).
}

based on serial kinematics. First the different mobile and largescale metrology systems that use optical position measurements are discussed. Hereafter different selection criteria for these systems are discussed. Finally, the successful application of optical measurement systems is illustrated in two industrial cases.

\section{Overview of different mobile CMMs}

There is a broad range of large-scale measuring systems that are working on different principles. The most common are summarised beneath:

\subsection{Measuring systems using two angles and one length}

Most of the large-scale measuring systems rely on the determination of one length and two angles (Fig. 1). Coordinates are initially measured in a spherical coordinate system $(\rho, \varphi, \theta)$ that can be translated to Cartesian coordinates $(x, y, z)$. For each system, the angles are measured by means of angular encoders. The length measurement differs for the different measurement systems and determines also the achievable accuracy. 


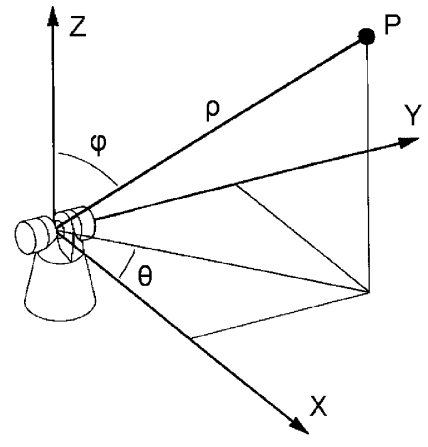

Fig. 1. Working principle of laser tracker, total station and coherent laser radar.

Laser trackers measure the length through interferometry. The interference of the reference beam and measuring beam determines the relative displacement of a retroreflecting target. This target is usually a spherical retroreflector. Since the measurement of the displacement is relative, the interruption of the beam implies that the retroreflecting target has to return to a reference position (nest). Current laser trackers are equipped with additional absolute distance meter (ADM) functionalities which enable (less accurate) absolute measurements. There are also laser trackers available with only ADM, which are currently performing almost as good as laser trackers with a built-in interferometer.

Total stations also measure distance with only ADM, but have no tracking functionality. Total stations can be programmed to search within a specific area for a retroreflecting target but are not able to follow a moving target in space.

Laser radar techniques enable to measure distances without the need for a retroreflector. A coherent laser radar needs a reflectivity of just $1 \%$ of the signal, and is able to measure directly on the surface of the object [1]. This makes it possible to scan many points in a short period of time and eliminates the compensation for the retroreflector radius.

\subsection{Measuring systems using multiple angles}

Instead of using two angles and one length, three angles can also define uniquely the position of a point in space.

Camera-based triangulation systems apply this principle. Three linear CCD cameras look at targets in space and each camera determines one angle and thereby one plane that contains the target. By using three cameras, the resulting position of the target can be calculated based on the three measured angles. This can be visualised as an intersection of three planes (Fig. 2). There are also systems that use two matrix CCD cameras instead of three linear CCDs. In this way four angles are measured instead of three. This redundancy enables to detect deviations from the precalibrated camera positions. Some of these systems work with fixed camera positions; others use movable cameras, which makes it possible to adapt the measurement volume and resolution depending on the measurement task.

The working principle of the fairly new $i G P S^{\circledR}$ system is also based on multiple angle measurements; however, the system setup is completely different. Different 'satellite' stations spread in the measurement area emit rotating planes of laser light under different angles, as illustrated in Fig. 3. Sensors are able to detect the time interval between the pulses of light. Based on this information it is possible to determine the horizontal and vertical angles of the sensor with a satellite. When the sensor 'sees' at least two 'satellites', the position of the sensor in space can be determined, assuming that the position of these satellites is precalibrated.

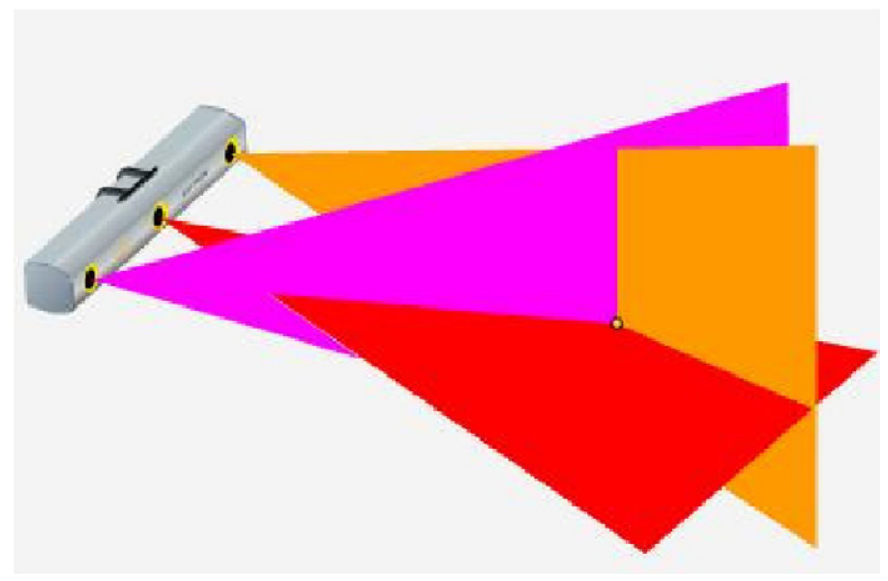

Fig. 2. Working principle of a camera-based triangulation system with 3 linear CCD cameras, resulting in 1 horizontal and 2 vertical angle measurements [2].

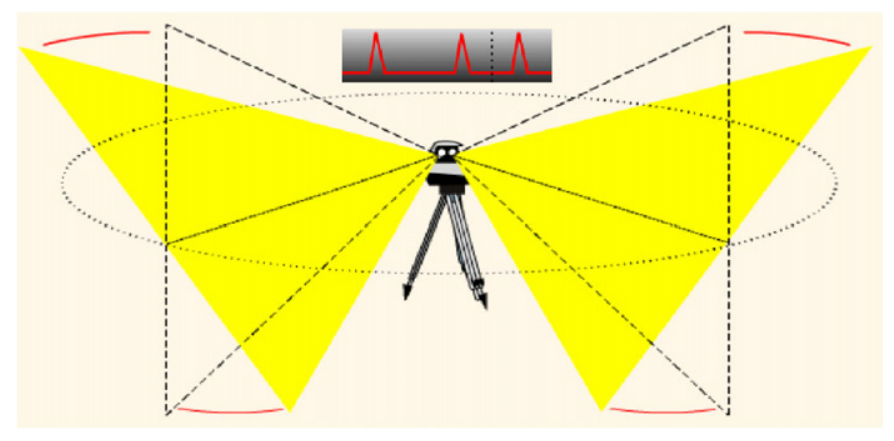

Fig. 3. The iGPS system emitting 2 rotating planes of laser light [3].

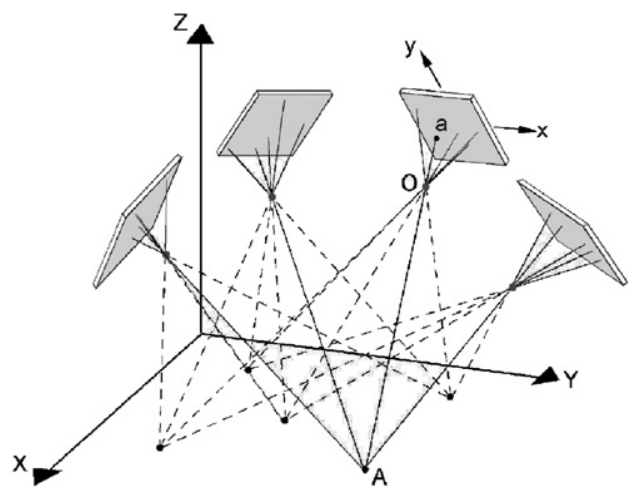

Fig. 4. Working principle of photogrammetry [4].

Photogrammetry is another large-scale measurement technique that, in fact, is based on angle measurements. With photogrammetry the position detection is based on different 2D images of multiple measurement targets as illustrated in Fig. 4. An important difference with the camera-based triangulation systems discussed above is the fact that the camera positions are not precalibrated. The camera positions are calculated afterwards together with the target positions. The technique of photogrammetry is already known for some decades but since the introduction of digital photography and powerful computers, the high degree of automation has enhanced its productivity. The position detection is based on targets mounted on the measurement object; so there is no need for a probing system. 


\subsection{Measuring systems using multiple lengths}

As it is possible to measure based on only angle measurements, it is also possible to measure based on multiple length measurements. This approach is sometimes used to overcome the lower accuracy of the angular encoders of laser trackers. The position of the target is then measured by multiple laser trackers or with one laser tracker from different locations. Only the (accurate) length measurement of the laser tracker is used. When enough points are measured from different locations, it is possible to calculate the tracker locations and the position of the measuring points. This principle is already used to calibrate conventional CMMs [5], in the domain of large scale metrology it is currently mainly used for research activities [6].

\section{Selection of mobile CMMs}

All different systems discussed above have their specific advantages and disadvantages. Therefore it is impossible to define one best system for all measurements; even for one specific measurement task it can be very difficult to find the most suited solution. Apart from the investment cost, the factors that can determine the selection of a coordinate measuring machine are summarised in Fig. 5. The influencing factors can be grouped into three categories: task requirements, part restrictions and environmental restrictions.

\subsection{Task requirements}

An important factor determining the measurement task is the desired accuracy. It is important to realise that the measurement uncertainties of most mobile CMMs are strongly non-uniform in space. It is possible to take advantage of this fact and to reduce the measurement uncertainty. E.g. when measuring the distance between two holes with a laser tracker, the tracker can be placed so that there is a very small difference in angle between the two positions. Then, the accuracy will be close to the laser interferometer accuracy. Reliability of the results is related to the possibility of the system to detect latent measuring errors. Systems that calculate the position based on much redundant information can give an estimate of the possible measurement error, like for example photogrammetry systems. The number of measuring points is also an important factor. When we want to measure, for example, only one length with photogrammetry, it will nevertheless be necessary to add additional targets and take multiple pictures depending on the distance. The location of the measuring points can be an essential parameter; since line of sight is often necessary, it is sometimes impossible to measure hidden points without displacing the measuring device. With many systems it is also impossible to measure into (small) holes. The number of measurements can be related to the number of times that the same object has to be measured or to the number of times the same measurement task needs to be repeated each day, week or month. This is also related with the measurement time.

\subsection{Part restrictions}

Dimensions of the part that has to be measured can be a restriction for the measuring system. Certain camera-based triangulation techniques have limited measurement volume compared with other systems. The material properties of the part to be measured can limit the application of a specific measurement system. With photogrammetry, for example, often magnetic targets are used; this is of course not possible when measuring parts in aluminium or plastics. Limited stiffness of the part implies that only non-contact techniques are applicable. Surface quality (e.g. very shiny surfaces) can restrict non-contact measurements with techniques like laser radar. Limited accessibility of the measurement point locations can, as stated above, also be a problem.

\subsection{Environmental restrictions}

The temperature is an important error source within the field of large-scale metrology. Not only temperature variations in time, but also temperature gradients in space are important [7]. It is important to know the susceptibility of the measurement system to temperature variations. Also the susceptibility to vibrations is system specific, which makes some systems less suited for heavy shop floor environments. Not all systems are suited for outdoor use. The available space can also be an important restriction. This is for example the case with photogrammetry systems, which need enough space around the object to take all the photographs. Air turbulences have an important influence on the propagation of light through air and limit thereby the achievable accuracy. This accuracy can also be limited through ambient lighting that disturbs the detected signal. Furthermore, the contamination of the optical equipment (like e.g. lenses) can lead to an important decrease of the accuracy.

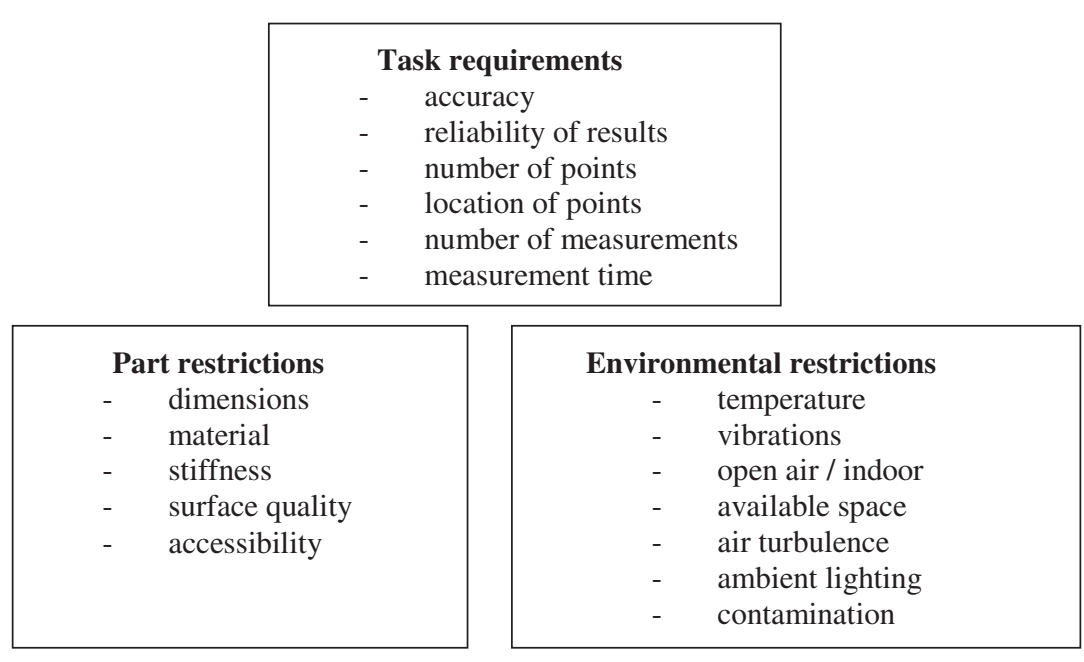

Fig. 5. Factors influencing selection of mobile CMMs. 


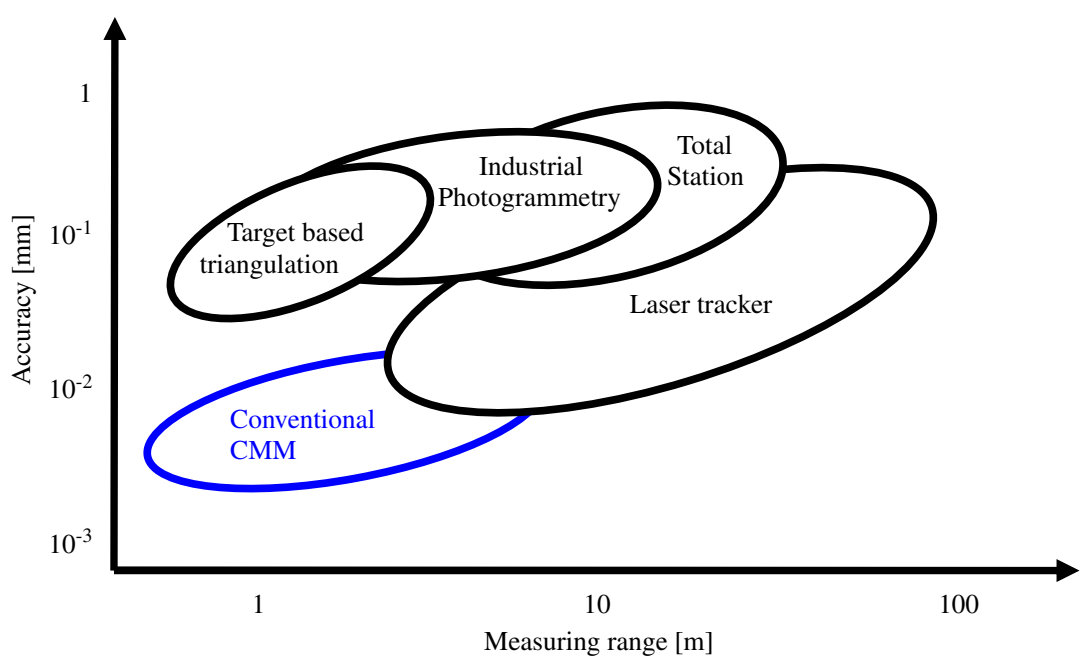

Fig. 6. Measuring range and accuracy of optical mobile CMMs in comparison with conventional CMMs.

\subsection{Selection}

Taking into account the topics discussed above, it is clear that selecting a suitable measurement device for a certain measurement task can be a difficult exercise. Therefore it is important to identify clearly your needs (task requirements) and your limitations (part and environment restrictions). Based on this information, a limited selection of mobile CMMs can be evaluated.

Common selection criteria for mobile and large-scale CMMs are the measuring range and the accuracy. Fig. 6 gives an indication of how common mobile measuring systems perform for these two criteria. It is just an indication since there are large differences between brands and accuracy specifications are difficult to verify due to lack of standards and the fact that the measurement uncertainty for mobile CMMs is highly task specific.

It is always a good practice to arrange some test case measurements with different systems in order to see how the systems perform before making the final selection.

\section{Case study: measurement of large casting objects}

\subsection{Problem statement}

This case study discusses the measurement of a large casting for a wind turbine gearbox. As the castings undergo a final machining process, it is important to know the amount of excess material. On the one hand, there must be sufficient material to remove in order to ensure that the outer layer of the casting, with many impurities, can be completely removed. On the other hand, too much material will only raise machining costs. The presence of sufficient excess material will be strongly determined by the alignment of the part on the machine. Nowadays, this alignment is done through trial and error. For large castings, costing thousands of euros, this is a very cost inefficient method. In addition, not each casting is the same; the casting patterns are subjected to wear and some parts are even produced by different foundries. That is why a method that enables a systematic entrance control of the castings is needed.

By measuring the castings before machining it is possible to determine in advance the most suited alignment and detect castings that deviate too much to fabricate good parts, so that at least the machining costs can be saved. This also

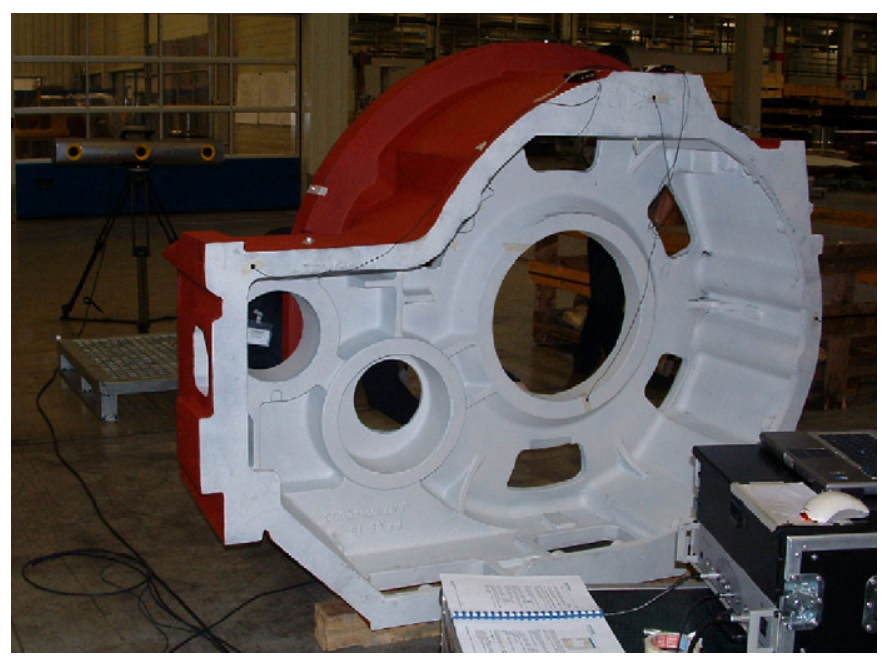

Fig. 7. Camera-based triangulation system in the back; casting in the front.

enables to give feedback to the foundries about the quality of the casting.

\subsection{Solution}

The most important task requirements for this measurement problem are a fast measuring time, and as much measuring points as possible, certainly on the surfaces that have to be machined. Important part and environmental restrictions are the dimensions of the part (approximately $3 \mathrm{~m}$ ) and the ability to measure in a shop floor environment. For this case study a camera-based triangulation system was used (Fig. 7). By looking with the camera system to the LEDs mounted on a probe the position of the probe tip in space can be determined (Fig. 8).

The part was also measured with a fringe projection system. Using this system took much more time to measure (because of the dimensions of the part), but it had the advantage of having a complete digitisation of the part.

\subsubsection{Preparations}

The castings were positioned in the range of the camera system. Dynamic reference LEDs (Fig. 9) were used in order to extend the range of the camera and to reduce the possible 


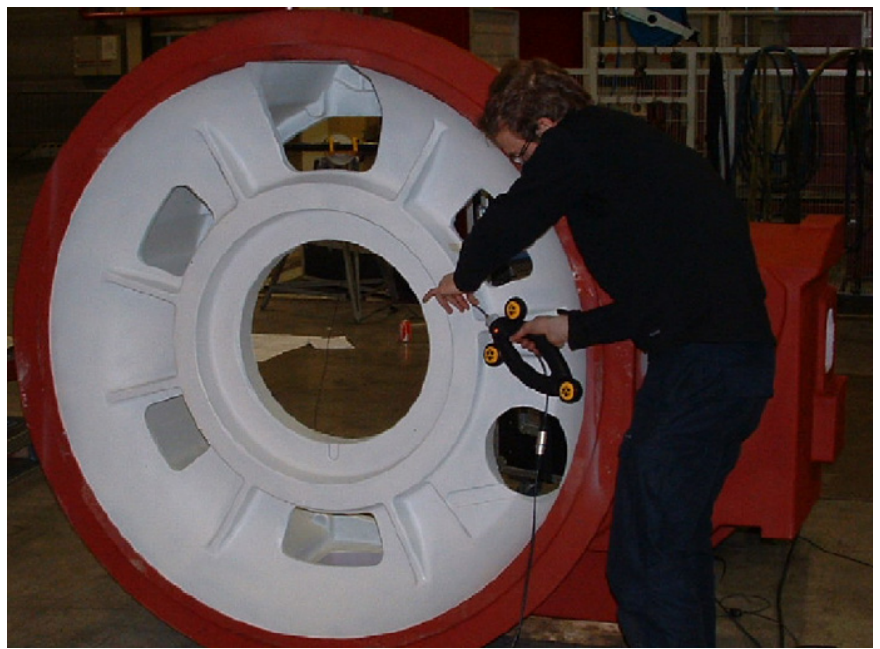

Fig. 8. Handheld probe with LEDs tracked by camera system.

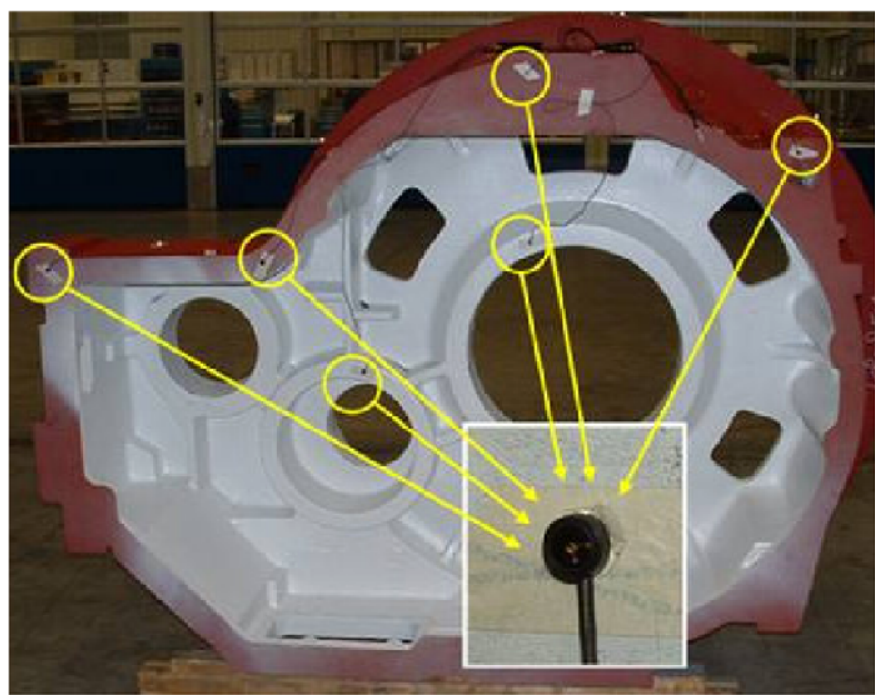

Fig. 9. Dynamic reference LEDs.

influence of vibrations. This is an important advantage of camerabased triangulation systems. Since it is possible to measure the position of multiple targets at once, it is also possible to track the movement of the part to measure. In order to measure the complete casting, front and back, internal cones (jump points) were placed on the casting (Fig. 10). These points are measured when the camera is on the front side and can be remeasured when the camera is placed on the back side, so measurement points from both sides are linked together.

\subsubsection{The alignment}

When we want to compare the measurement data with the nominal CAD file we need to align the measurements with the $\mathrm{CAD}$ file. This alignment is done in the same way as the alignment of the part on the milling machine. This has an important advantage for predicting problems: when there is a material shortage detected while comparing measurement results with the CAD data, problems will also occur while machining the part.

The $X-Y$-plane is determined by the reference plane (Fig. 11). The direction of the $X$-axis is determined by a line through the central and the outer hole. The projection of the centre of the central hole onto the reference plane is used to fix the translational degrees of freedom.

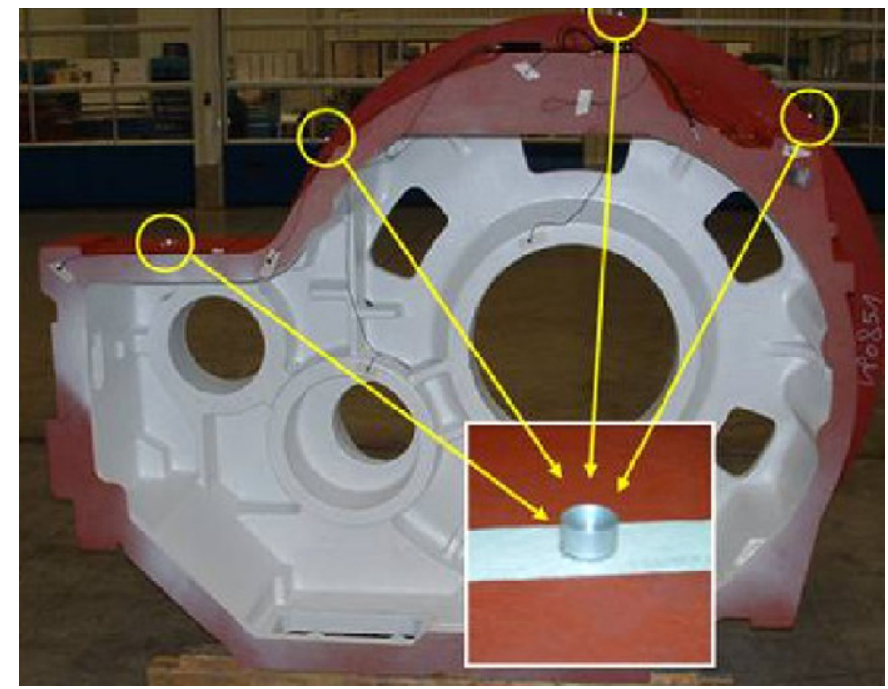

Fig. 10. Jump points.

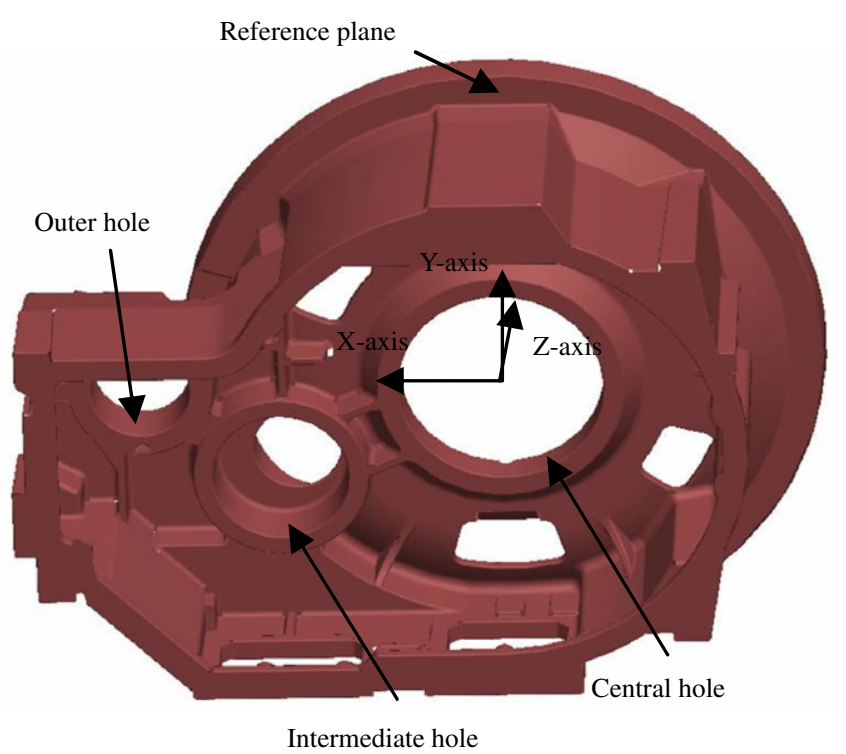

Fig. 11. Indication of the features used for the alignment.

\subsubsection{The measurement strategy}

Once the alignment is complete, points can be measured all over the object. Since the measurements are aligned with the CAD-file, deviations can be calculated immediately. These deviations are represented by coloured dots in Fig. 12. Green dots indicate that the material excess is within tolerances. Red dots represent a large material excess and blue dots a material shortage.

This material shortage is a consequence of too much shrinkage of the casting. This shrinkage can also be seen when the positions of the measured outer and intermediate hole are compared with the CAD positions. The outer and intermediate holes are shifted to the central hole as illustrated in Fig. 13. The consequence is a material shortage for the outer hole.

This problem can be reduced by shifting the CAD-file in negative $X$-direction, e.g. by making the centre of the measured intermediate hole coincide with the nominal centre. This workaround was sometimes used in production, but has the disadvantage that the problem is shifted to other regions that become critical. This is also confirmed by the obtained measurement 

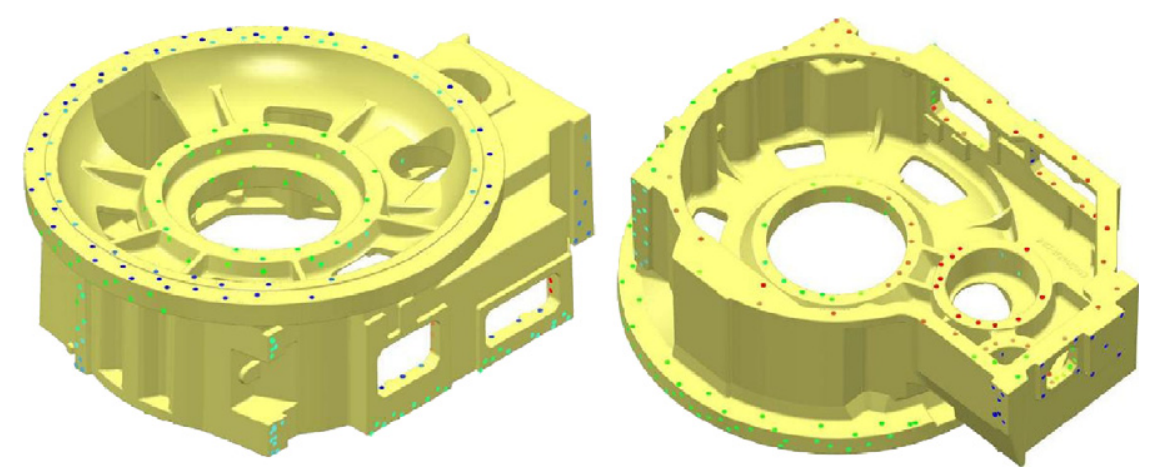

Fig. 12. View of the casting with coloured dots indicating the deviation from the CAD file of the casting.

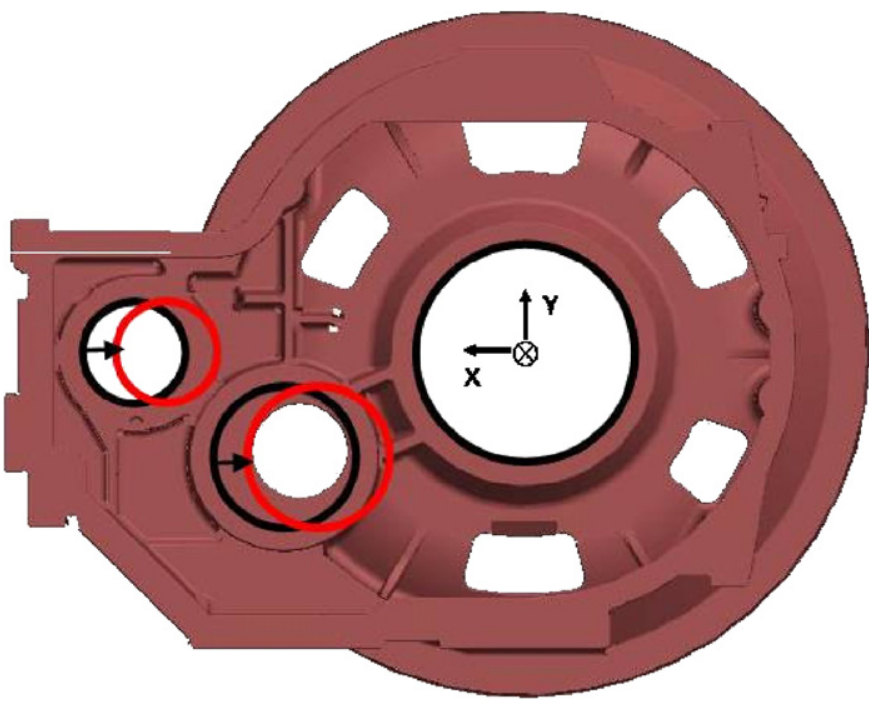

Fig. 13. Indication of the movement of the outer hole and the intermediate hole towards the central hole.

result, so it is concluded that shifting to the intermediate hole is not the most suitable solution.

In a last step, the measurement points indicating material shortage are isolated. These points are then least square fitted with respect to the CAD-nominal. During fitting, only translations were allowed. The result obtained with this method was considerably better than in the situations discussed above. Since only translations were allowed during fitting, the final shift in $X, Y$ and $Z$ directions can easily be transferred to the milling machine in order to distribute the excess material equally.

In fact even better results could be obtained if it was possible to use, instead of least square fitting, a fitting algorithm that maximises the minimal amount of excess material (minimax fitting). Unfortunately most measurement software does not support this type of fitting.

One has to keep in mind that the casting should meet the quality requirements in the first place, so that shifting of the machining alignment is not necessary. The proposed method makes it possible to detect problems before machining and (if possible) to adapt the alignment in order to overcome these problems.

A shortcoming of the proposed measurement method is the limited amount of measured points, so there is always a risk of not having measured important local defects. In that respect scanning methods have an important advantage. On the other hand the measurement time for these methods is often much longer, especially for large objects as these.
Fig. 14 gives the result of a scanning measurement with a fringe projection system (ATOS II, GOM). It is clear that this gives more information about the casting. The point cloud that is generated can also be used for further studies, for example reverse engineering, finite elements calculations, vibration analysis, etc. When only information is needed about the excess material in some specific areas, discrete point measurements are satisfactory and also faster.

\section{Case study: measurement of a double-decker train}

\subsection{Problem statement}

After a structure of a train is built, the structure is measured to be sure that all dimensions are within specification. In the manufacturing plant that is studied here, this is done, at present, with a total station and by one operator.

The task requirements are the following: approximately 100 measurement points at predefined positions, measurement time of less than $4 \mathrm{~h}$ and a measurement uncertainty of about $0.1 \mathrm{~mm}$. The most important task and environmental restrictions are the large dimensions ( $25 \mathrm{~m}$ length) and the location within the production line.

The current measuring time is $6 \mathrm{~h}$, which is too long to meet, increasing production rate. Another disadvantage of the current total station is the impossibility to measure when people are working on the structure to avoid measurement errors by vibrations. The goal is to implement a new measurement system that is able to reduce the measurement time to less than $4 \mathrm{~h}$. If possible, the system should also be less susceptible to vibrations.

\subsection{Solution}

For this case a photogrammetry-based measurement system is proposed. An important advantage of the photogrammetry technique is that the measured object need not be stationary between the measurements. A disadvantage of the technique is the space that is needed around the object to take the pictures and the large number of pictures that have to be taken from different orientations, especially for large objects like a train. The case study determines if this technique is suited for this particular measurement task.

\subsubsection{Preparation}

Encoded markers are placed directly on the structure of the train (Fig. 15) on the positions that need to be measured. These markers are not only necessary as a measuring point but are also used to link the different photographs with each other. Encoded 

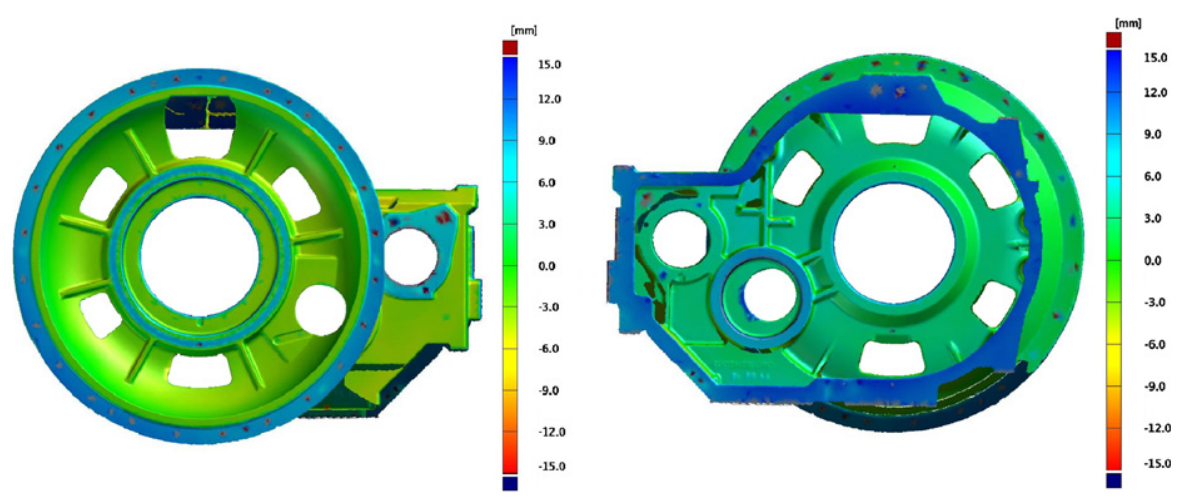

Fig. 14. Colour plot of a full scan of the casting compared with the CAD file of the machined part.

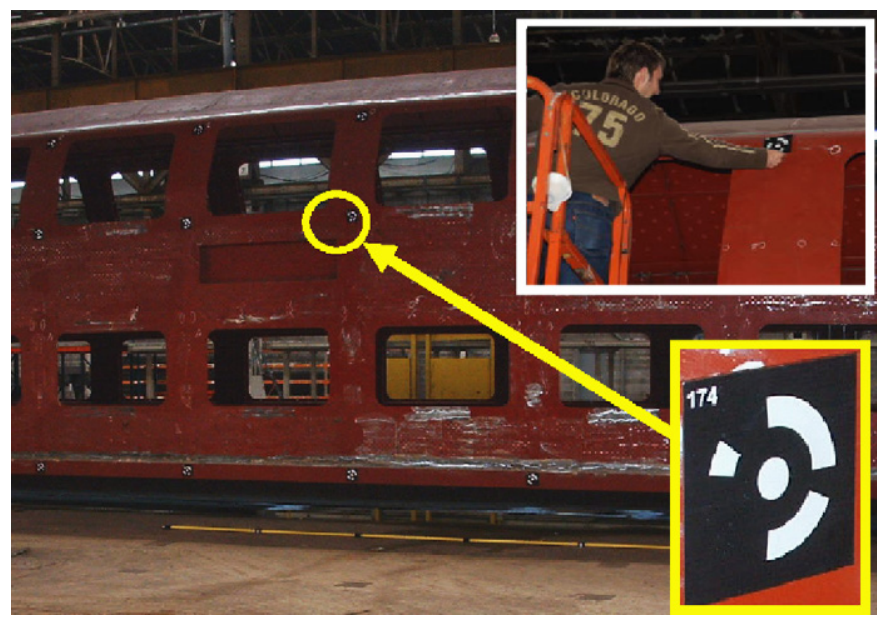

Fig. 15. Placement of encoded markers.

markers are used to make the analysis of the results easier by using macros. This means that each marker has a specific place on the train. The markers are magnetic so that they can be placed and removed easily.

The thickness of the markers is automatically compensated. Some encoded markers are placed on special adapters to indicate the centre of a hole or other features (Fig. 16). In this case the adapters are placed into the features that are used as the references for alignment.

For the scaling of length results an accurate reference standard is needed. In this case, two encoded reference standards of $5 \mathrm{~m}$ length were used. These are automatically recognised by the software. These standards were put next to the train (Fig. 17). If the standards were placed onto the train, it would even be possible to move the train during the measurements. One standard is strictly necessary, but by using two standards redundancy is added.

The actual setup of this case consists of approximately 120 points that have to be measured. Before starting the measurements, the camera needs to be calibrated. First the diaphragm and the focus distance of the camera have to be set. Afterwards four calibration images were taken. These images always include the reference standard and are rotated $90^{\circ}$ to each other (Fig. 18). The images are taken with a professional high-resolution digital camera and a $24 \mathrm{~mm}$ fixed lens. The images are sent wireless to the computer for processing.

\subsubsection{The measurements}

All measurement targets have to be photographed from different directions and in this case also different heights (Figs. 19
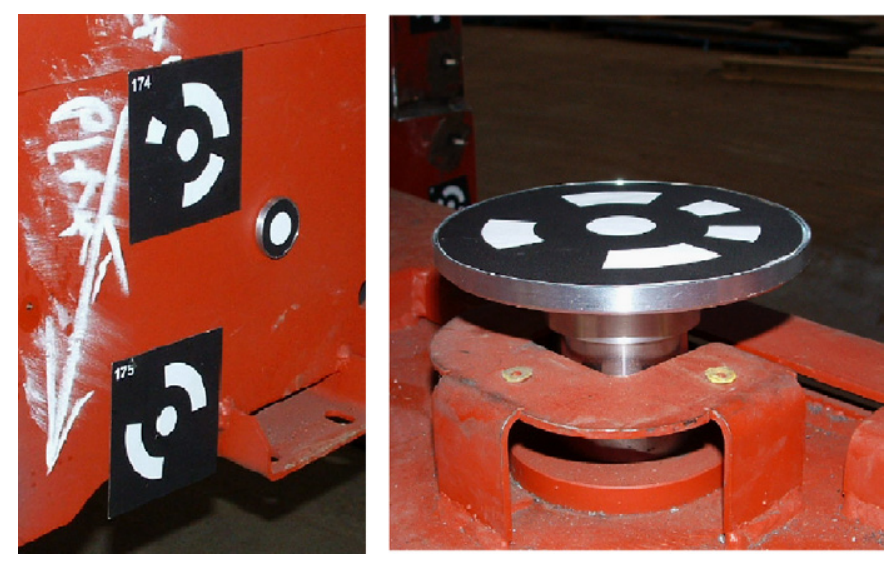

Fig. 16. Round markers and special adapters.

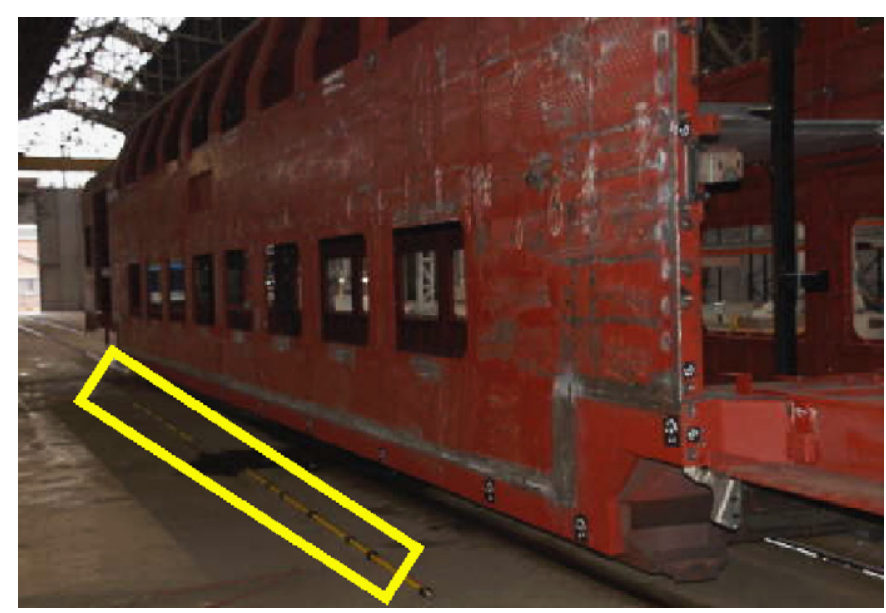

Fig. 17. Placement of encoded reference standards.

and 20). Each encoded marker is recognised automatically by the software (Fig. 21). A marker has to be visible in multiple images in order to be able to calculate its position. To capture all the measuring points, about 150 photographs are needed.

When enough photographs are taken the software bundles these images and, after scaling the results with the standards, all positions are calculated. Using these points, different features (like lines, planes, etc.) can be calculated as with conventional CMM software. Fig. 22 gives a screen shot of the captured data. The individual points can be used to calculate the deviations against nominal CAD data. Analysis of the result can be automated by the use of macro scripts and the encoded targets. 


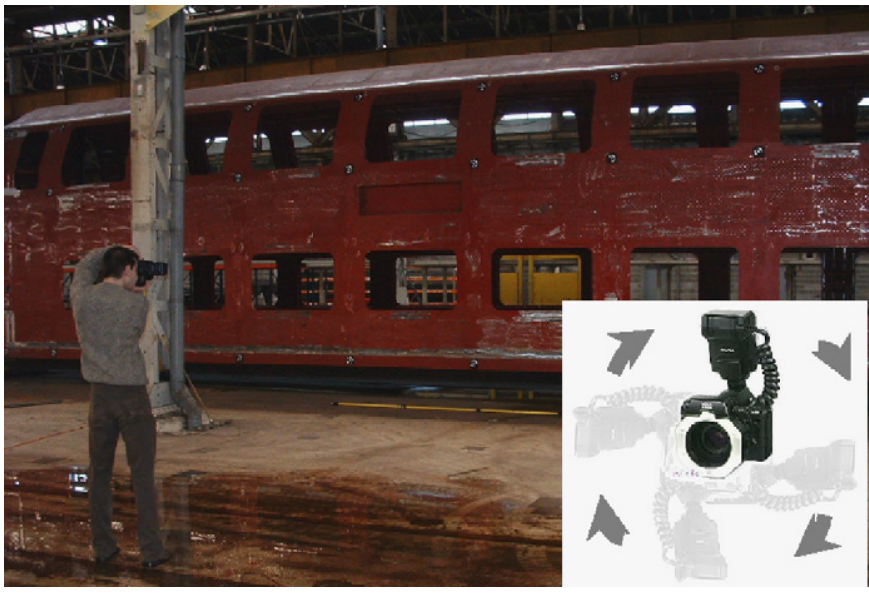

Fig. 18. Taking the four calibration images $90^{\circ}$ rotated against each other.

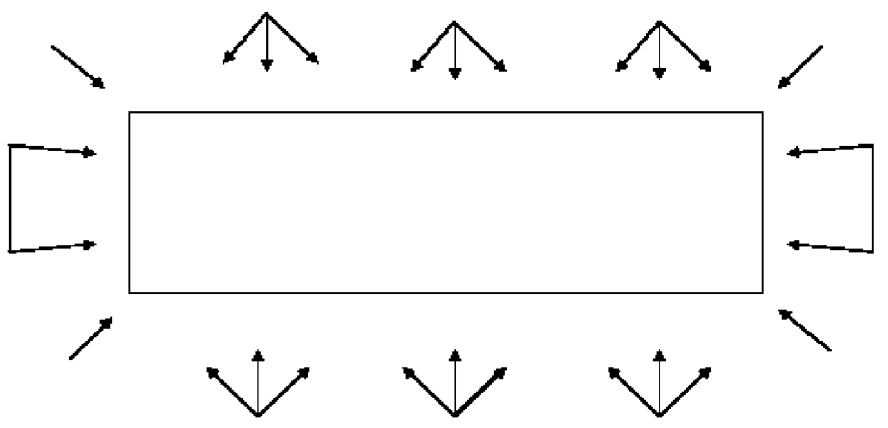

Fig. 19. A sketch of the camera positions and viewing directions.

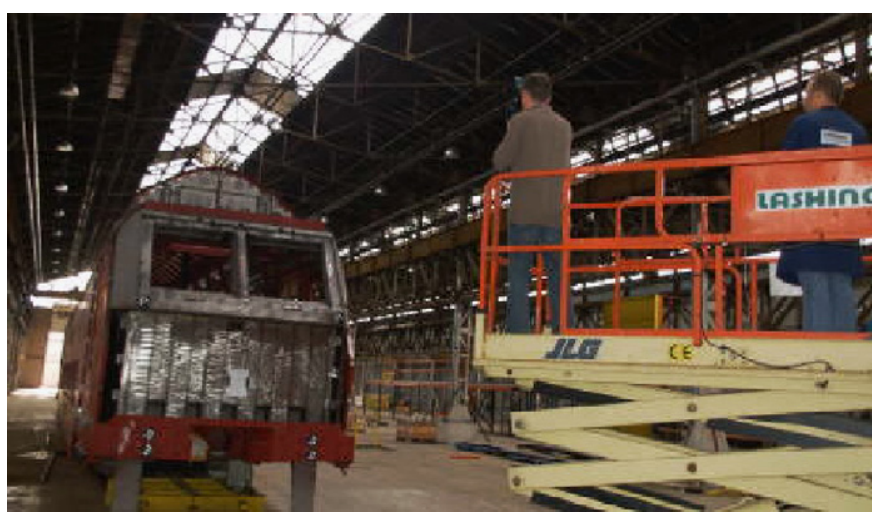

Fig. 20. Taking the pictures from different heights and directions.

\subsubsection{Time progress}

The objective was to get a total measuring time of $4 \mathrm{~h}$. This objective is certainly reached as can be seen in Table 1 . The measurement time could even be reduced to $2 \mathrm{~h}$, if the measurement is optimised.

\subsubsection{Accuracy}

The error on the two standards of $5 \mathrm{~m}$ after bundling and scaling was about $25 \mu \mathrm{m}$. This could be related to $25 \mu \mathrm{m} / 5 \mathrm{~m}$ or $125 \mu \mathrm{m}$ over a distance of $25 \mathrm{~m}$. The difference between the distance obtained by the total station and the distance obtained with the photogrammetry system was only $0.3 \mathrm{~mm}$ for the whole length $(25 \mathrm{~m})$ of the train.

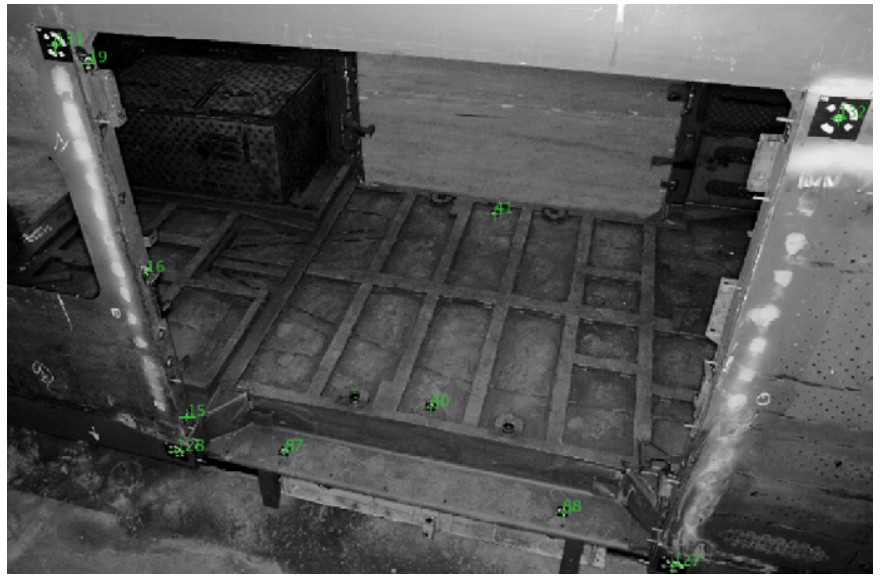

Fig. 21. The green label indicates the recognition of the encoded targets.

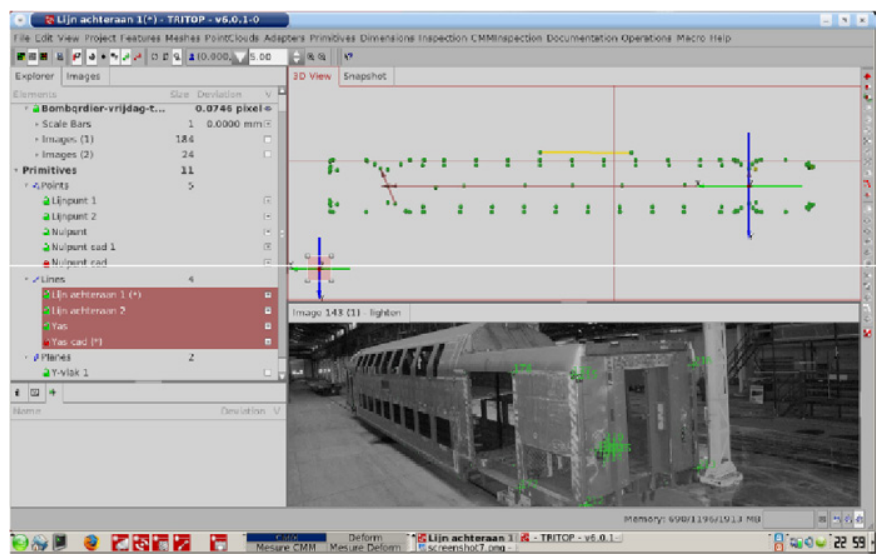

Fig. 22. Screen shot of captured data.

Table 1

\begin{tabular}{ll}
\hline & Time \\
\hline Preparation & $1 \mathrm{~h}$ \\
Measuring and analysing & $1 \mathrm{~h} 30 \mathrm{~min}$ \\
Remove markers & $20 \mathrm{~min}$ \\
Total time & $2 \mathrm{~h} 50 \mathrm{~min}$ \\
\hline
\end{tabular}

\subsubsection{Advantages and disadvantages}

The most important advantages and disadvantages of the photogrammetry system compared with the current total station are summarised below:

Advantages

- The object does not have to stay fixed.

- The system does not have many expensive and vulnerable parts.

- The preparation of the measurements (placement of the targets) can be done before the train arrives in the measurement station. So the actual measuring time can be limited to approximately $1 \mathrm{~h}$.

- The errors of bundling give an indication of the measuring errors and prevent making large measuring errors.

\section{Disadvantages}

- One has to place targets. This can be a problem when the object is not magnetic, thus no magnetic markers can be used. 
- There has to be sufficient available space around the object.

- One limited measurement (for example only the length of the train) could take just as much time as measuring the whole train.

- There is more need for adapters (e.g. for invisible points, diameter and centre of a hole).

\section{Conclusion}

This paper started with a discussion of some different optical measurement techniques for large-scale metrology. They were subdivided into three categories: systems that use two angles and one length measurement to determine target positions, systems that only use angles and others that only use lengths. Most commercial available mobile and large-scale metrology systems can be classified in the first two categories.

It is often difficult to choose the most suited measuring system for a specific measurement task. There is a wide range of systems that all have their own particular strengths and weaknesses. When selecting a mobile CMM, three elements should be kept in mind: task requirements, part restrictions and environmental restrictions.

Subsequently, two industrial case studies are discussed. In the first case study, a large casting is measured with a camera-based triangulation system. Results show that a systematic inspection of castings is an added value for the production process: by measuring the part it is possible to determine the most suited alignment for machining, so that the presence of enough excess material is guaranteed over the whole part. It was also possible to detect in advance bad castings, so that machining costs can be saved.

The second case study shows that, due to the recent developments in digital photography, photogrammetry becomes a valuable alternative for the measurement of large constructions. Previously, almost only total stations and laser trackers were used for these measurements. In this particular case it was possible to obtain the same accuracy as with a total station and to reduce the measurement time from 6 to less than $3 \mathrm{~h}$.

\section{Acknowledgements}

The authors would like to thank the collaborating companies, and also the Flemish Institute for Innovation through Science and Technology (IWT) for the funding of the project.

\section{References}

[1] White DA. Coherent laser radar: true noncontact 3-D measurement has arrived. Quality Digest, August 1999.

[2] Metris Optical CMM. Product CD, 2005.

[3] Arc Second. White Paper: error budget and specification, 2002.

[4] Estler WT, Edmundson KL, Peggs GN, Parker DH. Large-scale metrology-an update. Ann CIRP 2002;51(2):587-609.

[5] Schwenke H, Franke M, Hannaford J. Error mapping of CMMs and machine tools by a single tracking interferometer. Ann CIRP 2005;54(1):475-9.

[6] Hughes B. Large scale metrology at NPL. In: Large scale metrology workshop, Braunschweig, Germany; 2006.

[7] Puttock MJ. Large-scale metrology. Ann CIRP 1978;27(1):351-6. 